\title{
CORRECTION
}

Check for updates

Cite this: J. Mater. Chem. B, 2019

7, 346

DOI: $10.1039 / c 8 t b 90182 a$

rsc.li/materials-b

\section{Correction: Simultaneous and controlled release of two different bioactive small molecules from nature inspired single material}

\author{
Adil M. Rather, ${ }^{a}$ Arpita Shome, ${ }^{a}$ Bibhas K. Bhunia, ${ }^{b}$ Aparna Panuganti, ${ }^{a}$ \\ Biman B. Mandal ${ }^{b}$ and Uttam Manna*a
}

Correction for 'Simultaneous and controlled release of two different bioactive small molecules from nature inspired single material' by Adil M. Rather et al., J. Mater. Chem. B, 2018, 6, 7692-7702.

\begin{abstract}
The authors regret that the incorrect affiliation was noted for two of the authors in the original manuscript. The correct affiliation for Bibhas K. Bhunia and Biman B. Mandal is: Biomaterial and Tissue Engineering Laboratory, Department of Biosciences and Bioengineering, Indian Institute of Technology Guwahati, Assam 781039, India. The corrected list of the authors and their affiliations is as shown above.
\end{abstract}

The Royal Society of Chemistry apologises for these errors and any consequent inconvenience to authors and readers.

\footnotetext{
${ }^{a}$ Department of Chemistry, Indian Institute of Technology-Guwahati, Kamrup, Assam 781039, India. E-mail: umanna@iitg.ernet.in

${ }^{b}$ Biomaterial and Tissue Engineering Laboratory, Department of Biosciences and Bioengineering, Indian Institute of Technology Guwahati, Assam 781039, India
} 